\title{
Artificial Intelligence Versus Conventional Mathematical Techniques: A Review for Optimal Placement of Phasor Measurement Units
}

\author{
Kinfe Negash $^{1} \cdot$ Baseem Khan $^{1} \cdot$ Estifanos Yohannes $^{1}$
}

Received: 11 May 2016/ Accepted: 21 July 2016 / Published online: 8 August 2016

(C) Springer Science+Business Media Singapore 2016

\begin{abstract}
The Phasor Measurement Unit (PMU) is a contemporary metering device installed on system to enable the power system monitoring and control. PMUs are most accurate and advance time synchronized technology which provides measurements of voltages at the buses and also current phase values which are connected to those buses where these PMUs are located. PMU placement on each bus of a system is difficult to achieve either due to cost factor or due to non-existence limitation of communication facilities in some parts of the system. Moreover, as a consequence of Ohm's Law, when a PMU is placed at a bus, neighboring busses also become observable. This implies that a system can be made observable with a lesser number of PMUs than the number of busses. It is necessary to determine the minimum number of PMUs for entire observability of the power network. The optimal placement of PMUs (OPP) problem can be solved by various techniques. Hence in this paper a comprehensive review is presented on the OPP problems under the characterization of heuristic and mathematical methodologies. Also a comparison between
\end{abstract}

Baseem Khan

baseem.khan04@gmail.com

Kinfe Negash

kinfemichael.negash@gmail.com

Estifanos Yohannes

estif83@gmail.com

1 Institute of Technology, Hawassa University, Hawassa, Ethopia the various methods is also presented based on the various operation and cost related factors.

Keywords Phasor measurement unit - Conventional mathematical techniques - Artificial intelligence techniques

\section{Introduction}

The Modern deregulated electricity market is highly dependent on the real time information. A reliable, accurate, timely and complete set of measurement serves as a foundation for the secure real time grid operation. For this Monitoring and control of these grids in order to ensure reliable power deliveries are dependent on sophisticated information and control systems commonly referred to as SCADA/EMS systems, where system states estimation depends on unsynchronized measurement. These measurements have errors such as measurement and telemetry bias. To overcome these limitations in the SCADA, Wide Area Monitoring Protection and Control (WAMPAC) system is used. This system consist Phasor Measurement Units (PMUs) as fundamental components which gives synchronized and real-time voltages and currents phasor measurement. Global Positioning System (GPS) provides reference timing signals to achieve synchronization of sampling voltage and current waveform with respect to this reference time.

A PMU directly measure the voltage Phase of the bus where these PMUs are placed and also measure the current phases of a few or all the branches connected to that bus. In recent years rapidly increases use of PMUs to improve monitoring, needed to place these PMUs on all of the buses for full observability of the network. It is also impossible to place these units on entire system buses because PMUs and 
communication services are very costly. Thus determination of the optimal number of PMUs and its location for overall observability of the system is very important.

A proper methodology is required to find the optimum number of the PMUs which will fully observe the power network. To solve the Optimal PMUs Placement (OPP) problem a number of mathematical methods have been employed. Among these methods linear Programming, nonlinear programming, dynamic programming are the common optimization methodologies are proposed to solve this problem. Problems such as difficulties of obtaining local minima and handling constraints in conventional techniques are overcome by other optimization methodology like heuristic techniques, such as depth first search, iterated local search, simulated annealing, tabu search, genetic algorithms, differential evaluation, imperialistic, particle swarm optimization and ant colony optimization etc.

This paper reviews the research work and studies that have been done in the area of optimal placement of phasor measurement units (PMUs) that are presented to solve the typical optimal placement problem of PMUs. The formulation of this problem is described in Section "Optimal PMU Placement (OPP) Problem Formulation". The heuristic methods to solve the OPP problem are discussed in Section "Artificial Intelligence Techniques", the mathematical method is discussed in Section "Mathimatical Methods" and Section "Comparative Analysis of Mathematical and Artificail Intellegence Techniques" concludes this paper.

\section{Optimal PMU Placement (OPP) Problem Formulation}

PMU is an advanced measurement device installed at a bus (or node) of the system, measuring the voltage and current phasors of the line (or branch) it is associated with. Fig. 1 shows PMUs purely isolated to form a Wide Area Monitoring System (WAMS). GPS time stamped measurement signals are fed to a Phasor Data Concentrator (PDC) by using PMUs. The PDC collects and sorts the phasor measurements and signal processor converts data of PMUs into useful information which is visible on Human Machine Interface (HMI).The operator can easily access the critical information of the power system state. Some rules can be used for the placement of PMUs which are given in [26] like, assigning one voltage measurement at the bus where PMU is located, one branch current measurement, one voltage and current pseudo measurement.

The PMUs can be placed at planned buses to completely observe the total network. These located PMUs are measuring the voltage phase value of that bus and current phase values of the lines which are connected to the same bus. The

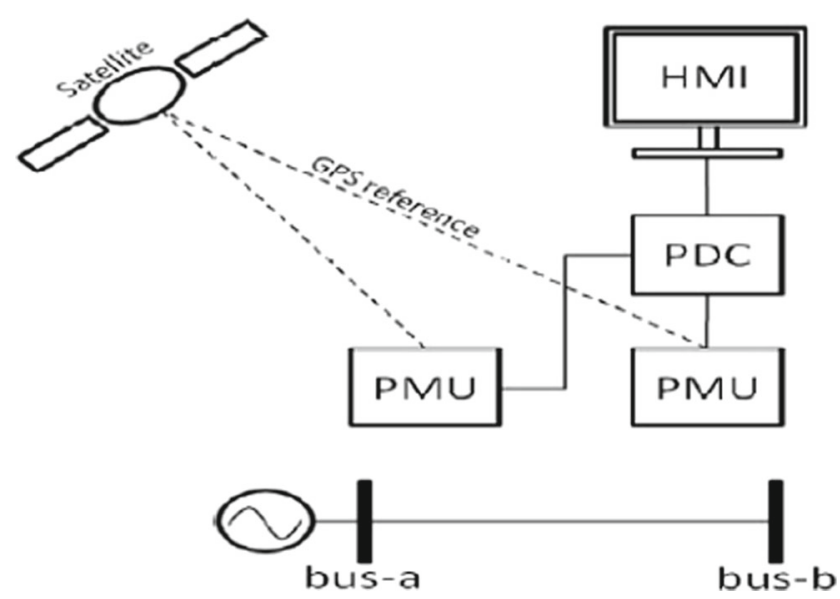

Fig. 1 Layout of PMU along with GPS time stamped signals [26]

aim is to completely observe the network with an optimum number of PMUs. The problem for n-bus system is formulated and solved by integer programming method [51] as given below:

$$
\begin{aligned}
& \min \sum_{i}^{n} W_{i} x_{i} \\
& \text { Subject to } f(x) \geq \hat{1}
\end{aligned}
$$

Where $x=$ binary decision variable vector

$i=$ bus number, whose entries are,

$x_{i}=1$ if a PMU is located at bus $i$,

$x_{i}=0$ otherwise.

$W_{i}=$ the cost of the PMU installed at the $i^{\text {th }}$ bus,

$f(x)=$ vector function, having non-zero entry if corresponding bus voltage is observable and

$\hat{1}=$ vector having all ones entries.

The nonlinear constraint expressions are created considering the placement and types of available measurements. Assume the phasor value of voltage at the bus where PMU located and values of current phasors along the branches which connected to that bus will be easily accessible. The other adjacent bus voltages will also be accessible. Determine the solution vector which is a set of minimum xi and satisfy above equation. The constraint function can be defined with the help of Binary Connectivity Matrix A which gives the information about bus connectivity of power network. The elements of matrix A is defined as,

$$
A(m n)=1 \text { if busm = busn }
$$

$=1$ if there is connection between bus $m$ and busn.

$=0$ Otherwise.

The constraint equations are considered for the three cases: (1) PMU measurements only, (2) PMU measurements and 
injections (i.e. zero injections) and (3) PMU measurements, injections and flows.

\section{Artificial Intelligence Techniques}

\section{Imperialistic Competition Algorithm (ICA)}

ICA is an evolutionary optimization algorithm which is inspired by socio-political process of imperialistic competition. This algorithm has proven its superior ability to achieve faster convergence and better solution compared to the other evolutionary algorithms.

In the proposed method in [1], the optimal placement of PMUs and minimization of the cost of associated CI (communication infrastructure) are carried out simultaneously by using ICA. That is to say the optimal placement of PMUs, the optimal location of the PDC and minimal communication paths between PMUs and the PDC are obtained simultaneously in order to make the network as fully observable as possible in terms of the state estimation.

\section{Cellular Learning Automata (CLA)}

Cellular learning automata (CLA) algorithm is based on the usage of learning automata (LA) to the state transition probability adjustment of cellular automata (CA). This method starts by specifying the internal state of every cell. Then, the reinforcement signal for each LA is determined on the basis of the rule of CLA. Finally, as for supplied reinforcement signal and action chosen by the cell, each LA updates its action probability vector. The desired state will be reached by continuing this process [85]. In [2] proposed a CLA algorithm to tackle the optimization problem, introducing new CLA local rules to enhance the optimization process in the formulation of a modified multi-objective PMU placement method by first calculating worth of observability for a set of PMUs in contingency, by ranking the prospective contingencies.

\section{Chemical Reaction Optimization (CRO)}

Chemical reaction optimization $(\mathrm{CRO})$ is a heuristic algorithm based on population processed by obtaining a lower energy stable state by simulating the action and reaction of molecules in a chemical reaction, which aimed to reach the minimum state of free energy. Authors in [3] proposed chemical reaction optimization (CRO) method to solve the problem of maximizing the power system observability by placing phasor measurement units (PMUs) in a multistage manner where the problem is constrained by the financial budgets available at each installation stage.

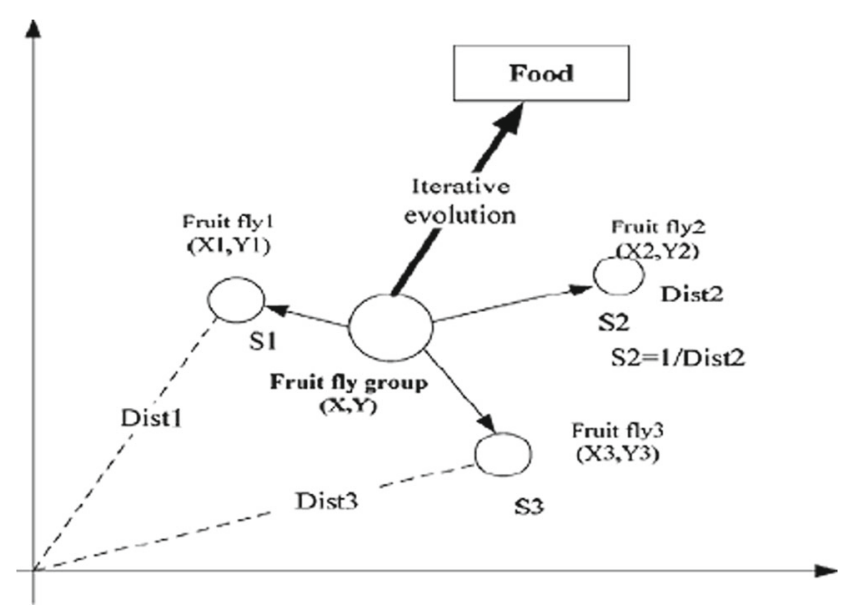

Fig. 2 the food finding iterative process of a fruit fly swarm [4]

\section{The Fruit Fly Optimization Algorithm (FOA)}

The fruit fly optimization algorithm (FOA) is a new method for finding global optimization based on the food finding behavior of the fruit fly. The fruit fly itself is superior to other species in sensing and perception, especially in osphresis and vision. The osphresis organs of fruit flies can find all kinds of scents floating in the air; it can even smell food source from $40 \mathrm{~km}$ away. Then, after it gets close to the food location, it can also use its sensitive vision to find food and the company's flocking location, and fly towards that direction too. Fruit flies are a kind of insect, which live in the temperate and tropical climate zones and eat rotten fruit [4]. Fig. 2 shows the food finding iterative process of a fruit fly swarm.

The authors in [4] develop a modified fruit fly method for solving PMU placement problems. This method is found to speed up the convergence.

\section{Genetic Algorithm (GA)}

Genetic algorithm (GA) is adaptive heuristic search algorithm that repeats the process of natural evolution. This process is used to generate solutions to optimization and also search problems. Modeling natural selection is the base of genetic algorithm (GA) which does not need any secondary functions such as derivatives computation. Some positive characteristics of GA which make it more usable in optimization problems are as follows:(a) probability of local minimum trapping is decreased,(b) computations of going from one state to another is declined, and (c) evaluation of the fitness of each string guides the search.

A genetic algorithm is proposed in [5] in order to minimize the number of PMU placement sites. The proposed method is numerical observability by using the minimum 
condition number of the normalized measurement matrix as criteria. The sequential elimination is used to find the essential measurements for the completely determined condition. The sequential addition is used to select the redundancy measurements under the contingency.

Reference [6] investigates the application of immunity genetic algorithm (IGA) for the problem of optimal placement of phasor measurement units (PMUs) in an electric power network. The problem is to determine the placement sites of the minimal set of PMUs which makes the system observable.

The authors in [7] presents a non-dominated sorting genetic-based algorithm (NSGA), which can successfully solve PMU placement problem with two competing objectives, such as minimization of the number of PMUs and maximization of the measurement redundancy. The optimization is performed without any preference information given with respect to the objectives.

In [8] genetic algorithms to find the optimal placement of PMUs has been generated using a MATLAB program .The genetic algorithm method was tested on three networktests, and the results were compared with other algorithms to prove that an optimal placement of PMUs ensures a total observability of the network and thus confirming the efficiency of genetic algorithm.

The genetic algorithm in [9] was used to calculate the best PMU location that meets the prespecified threshold of the LODE index for system observability reliability. In this work multiple solutions for optimal PMU placement in the normal system state are generated and the solutions are ranked using the observability reliability indices. Then, the best solution is compared with the optimal PMU placement solution considering the full observability under N-1 conditions of transmission branch failures. In [10] authors presents a genetic algorithm based method for optimal meter placement problem by which measurement system can be optimally determined and upgraded to maintain network observability. The proposed algorithm yields a measurement configuration that withstands any single branch outage and/or loss of single measurement, without losing network observability. In $[11,12]$ genetic algorithm (GA) is proposed for PMU and SCADA placement for state estimation identification. The results obtained in [11] are indicative of the fact that the optimal placement of PMU increases the accuracy of the obtained estimates and efficiency of the bad data detection algorithms. In [13] Genetic algorithm was developed to solve the proposed new model that utilizes network observability rules and determine the optimal investment decision for the placement of PMUs in the power grid. Reference [14] proposes an optimal PMU allocation method for substations based on a specialized Genetic Algorithm (GA) while in [15] the same method has been used for complete and incomplete observability. The authors in
[16] presented a new optimization algorithm for optimal PMU configuration based on combination of graph theory and genetic algorithm. The method for PMU allocation has shown a decent result, obtaining the same or smaller number of PMUs to fully observe the power system compared with the references. What is more important is that the computation cost is greatly reduced, which mainly attributes to the topology constraint analysis.

\section{Greedy Algorithm}

The greedy algorithm is an optimization methodology that follows the problem solving heuristic of making the locally optimal choice at each stage with the hope of finding the global optimum.

In [17], Optimal PMU placement problem was considered in the power system to improve the state estimation results. The PMU placement problem has been formulated as an optimal experiment design problem, with a class of well-known optimality criteria. It has been showed that the design criteria are usually related to a key property, sub modularity, which often allows efficient greedy algorithms. A greedy PMU placement algorithm that has been showed achieving an approximation ratio of $\left(1-\frac{1}{e}\right)$ for any PMU budget $\mathrm{K}$, which is the best guarantee among polynomial-time algorithms has been presented in [18]. In [19] a greedy algorithm is proposed to find unique optimal PMU placement with the best state estimation performance in three-phase network. A demonstration that the greedy algorithm is efficient and taking PMU phase mismatch into account will help in the improvement of the PMU placement was presented in [20].

\section{Differential Evolution (DE)}

Differential evolution (DE) concept employs N-dimensional element vectors to minimize ongoing space functions. Mutation, crossover, and selection are the principle operators utilized to carry out the global optimization. This heuristic method could be widely used in different cost function problems such as non-differentiable, non-linear and, multi-modal functions. Parallel computations, easy usage, and good convergence properties are other benefits of this approach [85]. In [21] authors presents a multi-objective optimal model of PMU placement with the objectives of minimization of the number of PMU and maximization of the $\mathrm{N}-1$ measurement reliability by using DE algorithm.

\section{Particle Swarm Optimization (PSO)}

Particle swarm optimization (PSO) is a population-based stochastic optimization technique in which each potential solution (called a particle) is assigned a randomized 
velocity and then flows through the problem hyperspace. PSO has been found to be extremely effective in solving a wide range of engineering optimization problems [22, 83].

A modified discrete binary version of particle swarm algorithm was used in $[21,25]$ as an optimization tool in finding the minimal number of the required PMUs for the complete system observability. At first, the PMU placement was solved with the goal of minimizing the total number of required PMUs for the complete system observability. Then, the effect of PMU loss or a branch outage was taken into consideration, and a placement scheme was obtained which maintains complete sys-tem observability during the contingency conditions. In [23, 24] PSO optimization is also used for obsevability with the objective of minimum cost of PMU.

\section{Iterated Local Search (ILS)}

Searching a smaller subspace, which is defined as local optima, instead of the whole solution space, is the main viewpoint of iterated local search (ILS) method. By utilizing an embedded heuristic, a sequence of solutions is provided in which the best solution is obtained if one were to utilize repeated random trials of that heuristic. The Iterated Local Search (ILS) metaheuristic in $[26,28,31]$ is used to minimize the size of the PMU configuration needed to observe the network .The algorithm is tested on IEEE test networks with 14,57 and 118 nodes and compared to the results obtained in previous publications.

In [29] authors use a local search heuristic method to derive the minimum number of PMUs. In addition to the practical model, this study also considers the ideal model, in which all load nodes are assumed to be zero injection. The numerical studies on various IEEE power test systems demonstrated the superior performance of the proposed algorithm in both the models in regard to computational time and solution quality. In [30] the empirical observability gramian is applied to quantify the degree of observability of a power system under a specific PMU configuration and an optimal PMU placement method for dynamic state estimation is proposed by maximizing the determinant of the empirical observability gramian. It is effectively and efficiently solved by the NOMAD solver which implements the Mesh Adaptive Direct Search algorithm.

\section{Intelligent Search Technique}

An intelligent-search technique called best first search (BFS) method utilizes information about each node by computing an evaluation function at each of them while moving towards the goal state. It helps the BFS algorithm to search and explore only the most promising nodes, thereby making the search process, simpler and faster one. The most promising nodes are identified as those having least/highest score of an evaluation function. While searching towards the least optimal cost path, the BFS algorithm has the capability to change its search path from the current search path to the most promising path (Kumar, 2008). This characteristic makes the BFS method superior over other graph theoretical methods for optimal PMU placement problem.

The authors in [33] utilize this method for the optimal placement of PMUs to make the power system topologically observable. The proposed method determines the optimal locations of PMUs in two stages. Stage I determines the suboptimal locations of PMUs using best first search, which is an intelligent search algorithm with an ability to change its search path from the current node to the most promising node. From the results of stage I, the redundant PMU locations are identified and eliminated in stage II using pruning. The proposed method is extended to take into account the presence of conventional flow measurements in the system. It is also found to be effective in handling both single as well as multiple flow measurements connected to a bus.

\section{Simulated Annealing (SA)}

Simulated annealing (SA) is a procedure for solving complicated combinatorial optimization in which the current solution is randomly altered. The new solution is the worse alteration with the probability that is reduced as the computation proceeds. An optimal solution for a large combinatorial optimization problem needs a fit perturbation mechanism, cost function, solution space, and cooling schedule to be solved by SA. Sufficiency of SA can be found by searching a large-scale system and obtaining good speed in terms of finding an optimal or near-optimal solution [84].

In [34] an algorithm dealing with communication constrained PMU placement is modeled within the framework of simulated annealing. Here the pragmatic constraint of not placing PMUs at buses without communication support is recognized.

The proposed method in [35] considers project practical view. It used the concept of indirect observation, the MST and SA method. And it also improved the state estimation with SCADA/EMS measurement and PMU measurement. The method has been declared as effective, and it also have some advanced points such as strong maneuverability and small investment.

\section{Tabu Search (TS)}

Tabu search (TS) is an adaptive algorithm that utilizes many other methods such as linear programming algorithms and heuristic concepts. This procedure is presented to solve the combinational optimization problems in scheduling and 
covering. Tabu list which is one of the main elements of TS consists of the number of recently visited states plus a number of unwanted states. Other main elements of TS are aspiration, diversification, and definition of a state and the surrounding area. There is a reset in TS when it is not converging [84].

A TS method to obtain a solution for the OPP problem by checking network observability with a numerical algorithm is introduced in $[36,37]$. The impact of three different TS initialization schemes, together with the two TS parameters (TL and TSI), on the optimal solution is investigated. The experimental results verify the superiority of the proposed RTS method over the MTS method. The effectiveness and flexibility of the proposed scheme is demonstrated by the simulation results tested on the four different IEEE test systems.

Now in the next section a review of mathematical techniques used for optimal placement of PMU is presented.

\section{Mathimatical Methods}

These methods are classified into three broad categories: Exhaustive search, Integer Programming, Integer Quadratic Programming (IQP).

\section{Exhaustive Search}

Exhaustive search is a general optimization technique that systematically enumerates all possible candidates for the solution and selects the candidate that satisfied the constraints at the optimum value of the objective function. Its main advantage is that it guarantees the finding of the global optimum. However, it is not suitable for large-scale systems with huge search space [84].

An exhaustive binary search method is implemented in [38] to determine the minimum number of PMUs needed to make the system observable. In case there is more than one placement set having the same minimum number of PMUs, a method is proposed to select the one resulting in the most preferred pattern of measurement redundancy. Due to its exhaustive nature, the method gives the global optimal solution, and hence the results for a number of standard test systems are reported to provide benchmark solutions for researcher's investigating various methods of optimal PMU placement.

A contribution in [27] is the development of an exhaustive binary search algorithm to determine the optimal placement of PMUs as well as calculate the channels availability, the availability of measurement system of each PMU placed, and the global avail ability of measurement and maximize the average identification capability of multiple line outages in [39].

\section{Integer Programming (IP)}

Integer programming (IP) is a mathematical programming method of solving an optimization problem having integer design variables, while the objective function and the constraints are linear, nonlinear, or quadratic, thus leading to integer linear programming (ILP), integer nonlinear programming (INLP) and integer quadratic programming (IQP) algorithms, respectively[49, 79, 84].

In [40] an IP was used to achieve optimal PMU placement for observability in a manner that can also detect and eliminate bad data in critical and critical measurement pairs, which otherwise can render the state estimation problem unsolvable or bad data undetectable. In [32], a new method is proposed for optimal PMU placement in power systems suffering from random component outages. The original ILP method is valid to get a PMU placement solution which can make the whole system topologically observable with minimum number of PMU.

In [41] authors proposed a new algorithm to determine optimum number of PMUs for fully observability of the system under any contingency. The suggested algorithm operates in four stages; the essential measurement for totally known condition is found out in first stage. In next stage, it selects redundant measurement set under any contingencies. Above both stages uses least condition number of normalized measurement matrix to select the proper measurement location. Further, minimum redundant measurement set is chosen from these measurements using the binary integer programming technique. Finally these measurements i.e. essential and minimum redundant measurements are arranged such that locations of PMUs are minimum. For this purpose heuristic method is used. The IEEE test systems results show proposed placement method which ensures the system observability under the given contingency. Also it provides the reliable measurement to the system.

The contributions of [42] by using ILP are: new OPP model is devised in which dc lines are modeled for observability analyses. The new OPP model seeks the minimal cost of the PMU placement while the cost of each PMU is a function of its measurement channels. This practical assumption is emphasized in our optimization model by the allocation of more than one PMU to a single node because a PMU would not have many channels due to technical limitations. The performance of the proposed model is examined on the IEEE test systems and a real-size power system. An Integer Linear programming is used to determine the optimal number and location of PMUs to make the system measurement model observable and thereby it can be used for power system state estimation. When installing PMUs in a power system that already has SCADA measurement units, it was suggested that the minimum amount of PMUs first be 
calculated and then install those PMUs in phases, where in each phase a certain number of PMUs are installed in locations providing the highest possible level of obsevability chosen from the optimal locations [43, 44].

In [45], OPP problem is solved using Integer Linear Programming considering sensitivity analysis. As the cost of one PMU is very high, so it is required to place minimum number of PMU's without neglecting the safety and security of the system. So the most sensitive buses in given power system based on load changes, are found and PMU's are optimally placed such that all the sensitive buses are observed. A technique is proposed in [46] for placing the PMUs in multiple stages over a given time period that ensures complete power system observability even under a branch outage or a PMU failure. The proposed technique is based on integer linear programming (ILP) and a multi-criteria decision-making (MCDM) approach. Integer Linear Programming method is used in first stage to find the optimum number of PMUs for the complete observability of the system. These PMU placements include the contingency cases such as single branch outage and single PMU outage. A multi-criteria decisionmaking (MCDM) subsequently developed to prioritizing the optimal PMU locations. This criteria include three indices, viz. tie-line oscillations observability index (TOI), voltage control area observability index (VOI) and bus voltage observability index (BOI). Finally, select the PMU locations in each stage by ranking the optimal locations using the proposed indices in the MCDM process. he number of PMU's are further reduced and hence reducing the system cost.

The authors in [47] addresses the various aspects of optimal PMU placement using binary integer linear programming(BILP). Cases with and without zero injection buses have also been considered. Minimum PMU placement problem may have multiple solutions, therefore, two indices viz. BOI (Bus Observability Index) and SORI (System Observability Redundancy Index) have been proposed to further rank these solutions. An integer-linear programming (ILP) model is developed in [48] to find the minimum number and location of synchrophasors subject to the feasibility of proposed backup protection scheme.

In [50] authors present an optimization model to determine optimum number of PMUs in power network. The proposed model consists of observability necessities based on a set of probabilistic criteria. The nonlinear mathematical expression related with the probability of observability index is changed to a linear model by a useful linearization technique. This linear model further described as a Mixed Integer Programming (MIP) problem. This proposed mathematical model is therefore well-suited with the Mixed Integer Programming solution tools and also it tested on large scale power systems.
A different formulation which is numerical and uses integer programming allows easy analysis of network observability [51]. An efficient and comprehensive formulation based on mixed integer linear programming method for the optimal placement of PMUs taking into account the channel avail-ability and different contingency conditions associated with power systems, i.e. single line outage and single PMU loss were also considered in [52].

In [53] mixed integer linear programming (MILP) framework was formulated for the proposed model that simultaneously optimizes two objectives functions: (i) minimizes the number of PMUs and (ii) maximizes the expected value of system's redundancy (or minimizes the un-observability of the system). Incorporating the impact of zero-injection buses, the formulation is used to evaluate the probability of un-observability of buses resulted from line outages and PMU loss.

An Integer linear programming based method for PMU placement has been considered to ensure complete system observability under critical contingencies. In case of optimum placement difficulties two indices have been proposed to choose the optimal solutions; namely Bus Observability Index (BOI) which gives the total number of PMUs observing a given bus and to get the sum of all BOI for the system, System Observability Redundancy Index (SORI) is used. An optimal PMU placement scheme is also be proposed considering different PMU channel capacities. This has been also indicated to help the power system planner to greater extent from application-cost point of view for PMU-PDC installation in the WAMS based power network at the planning stage. In addition to the cost factor, different criteria are suggested for the proper allocation of PMUs in a given system. Network observability, state estimation accuracy and robustness present samples of such criteria [54-60].

In [61], authors proposed Integer Linear Programming with a fast and novel practical model to determine the optimum number of PMU. It consist power system contingency conditions i.e. line outages and measurement losses. The proposed model is flexible than existing model because other situation can be included separately or all together in power systems. Also, model consist measurement limitations i.e. communication constraints of power networks. The main importance of this method; it take low time getting the result also it gives local optimal solution for large-scale power.

The problem of PMU placement is formulated and solved as an IP problem [62]. The solution will provide the minimum number of strategically located PMUs that will eliminate measurement criticality in the entire system. This implies that any bad data appearing on any single measurement will be detectable. It is shown that depending on the measurement configuration and the system topology, this 
goal can be achieved by using only a few extra PMUs to transform several critical measurements into redundant ones in the system.

A new multi-stage PMU placement approach to maximize the network observability during time horizons was proposed. Linear Integer Programming (LIP) is widely used to solve the OPP problems. An optimal placement of synchronized phasor measurement units (PMUs) to ensure complete power system observability in the presence of nonsynchronous conventional measurements and zero injections is also presented.[63-65].

A Binary Integer Programming technique for instantaneous PMUs placement and conventional power flow measurements for fault observability in power systems proposed in [66]. The formulation is initially based on a nonlinear programming problem is unsolvable therefore initial formulation results in a binary non-linear integer programming problem that is transformed into a corresponding ILP problem through Boolean implications. The transformation provides a high scale of scalability.

In [67] using the Binary linear Integer Programming method, local redundancy of the existing measurement is considered in the PMU placement strategy. Besides considering the number of PMUs implemented yearly limited, a multistage placement strategy is proposed according to the improvements on local redundancy.

A new method has been proposed in [68] that approached the PMU placement problem with purpose to ensure that all WAMPAC applications attain complete measurement observability. It presented an optimal method of placing PMUs for three categories of WAMPAC applications. The author weighted the application by all three criteria and categorize by the type of measurement shared. Category 1 consists of applications that require phasor measurement relative to a network's stability. Category 2 requires a bus' normal voltage and 3 requires measurement under faulted conditions. The phasing strategy ensures that initial PMU placements enable Category 1 and subsequently 2 and 3 .

In [69] authors propose a new PMU placement algorithm, which can minimize the number of PMU, considering network connectivity, ZIB, N-1 line contingency, system reliability and voltage stability level. By introducing the concept of system reliability, the proposed method can effectively reduce the number of PMU while ensuring the observability for major contingencies. The proposed method also employs the Voltage Stability Index (VSI) to weigh the priority level of bus, which is used to screen the severer buses during contingencies.

Based on the integer linear programming formulation proposed for optimal PMU placement, the authors in [70] presents a generalized integer linear programming formulation for cases including redundant PMU placement, full observability and incomplete observability. The proposed formulation improved the non linearity with and without conventional power flow and power injection measurements.

In [71] outages and system states is identified with the help of optimal sensors locations. In [72], an optimal phasor measurement unit (PMU) placement model considering power system controlled islanding so that the power network remains observable under controlled islanding condition as well as normal operation condition has been proposed using ILP. An ILP model for the optimal multistage placement of PMUs is proposed in [73]. The approach finds the number of PMUs and its placement in separate stages, while maximizing the system observability at each period of time. The model takes into account: the available budget per stage, the power system expansion along with the multistage PMU placement, redundancy in the PMU placement against the failure of a PMU or its communication links, user defined time constraints for PMU allocation, and the zero-injection effect.

In [74], an integer linear programming (ILP) framework for the optimal placement of phasor measurement units (PMUs), in the presence of conventional measurements is presented. Furthermore, by the proposed method, the power system is to be remaining completely observable during all possible single contingencies for lines and measurement devices. In [75] authors proposed a technique based on singular value decomposition (SVD) to define the role of each placement in dynamic stability assessment and find the best PMU places to achieve this goal. Since observability is still a very important factor, the integer linear programming method is used to solve the optimal PMU placement problem for full system observability. The contribution of the authors in [76] is to present a PMU placement methodology with observability redundancy; that is, to get more observability times of buses with the same number of PMUs. The method employs MILP, to make it possible to explore the global optimal solution unlike nonlinear programming.

The aim of the authors in [77] is to recognize the effect of channel capacity of PMUs on their optimal placement for complete power system observability. Initially, the conventional full observability of power networks is formulated. Next, a modified algorithm based on integer linear programming model for the optimal placement of these types of PMUs is presented.

In [78] authors presents a novel integer linear programming approach, which is shown to be equivalent to the ES-based PMU placement. The linearity of circuit equations ensures that there is a one-to-one relationship between the solutions of the proposed equivalent integer linear programming method (EILPM) and those of ES. Therefore, impractical ES is translated into EILPM, which can be 
solved in a few seconds or minutes. Furthermore, additional constraints for observability preservation following single PMU or line outages can easily be developed in the proposed EILPM. Moreover, the limitation of communication channels is also handled by rendering the nonlinear terms into linear ones.

\section{Branch and Bound Method}

In [86] authors presented the optimal positioning of the phasor measuring units for complete monitoring of power network in Khouzestan province in Iran. The (ILP) integer linear programming based branch and bound method is used to find the optimal number and position for PMUs. In [87] authors proposed an optimal phasor measurement units (PMUs) placement algorithm for power system observability and also increasing the performance of secondary voltage control scheme. The OPP is formulated to minimize the number of PMU installations subject to full network observability and monitoring pilot buses of the system to improve secondary voltage control performance. The branch and bound optimization method is used to solve the OPP problem which is suitable for problems with integer and boolean variables.

\section{Integer Quadratic Programming (IQP)}

Quadratic Programming (QP) is solving the optimization problem which has quadratic objective function and linear constraint. In this technique all design variables take only an integer value [85]. An integer-quadratic-programmingbased method in [80] is used to determine the minimum number and the optimal locations of the PMUs to ensure complete topological observability of the system. In [81] an integer quadratic programming approach is used to determine the optimal locations of PMUs. The optimization process tries to attain dual objectives: 1) to minimize the number of PMUs needed to maintain complete observability of the system for normal operating conditions as well as for the outage of a transmission line or PMU and 2) to maximize the measurement redundancy at all buses in the system. The method was applied on IEEE test systems considering the outage of a single transmission line or a single PMU. The proposed method can be used to determine PMU locations when conventional measurements, such as line flows and power injection measurements, are available. While the authors in [82] presents a new method for optimal placement of phasor measurement units (PMU) for complete power system observability by formulating the optimal PMU placement (OPP) problem as a quadratic minimization problem with continuous decision variables subject to nonlinear observability constraints. The optimal solution is obtained by an unconstrained nonlinear weighted least squares (WLS) approach.

\section{Comparative Analysis of Mathematical and Artificail Intellegence Techniques}

\begin{tabular}{lll}
\hline S. & Properties & Mathematical Technique \\
No. & & \\
\hline 1. & Problem size & Mathematical models are typically \\
& & used to model a system when the \\
& system is not so complicated. Some \\
& complex problems have defied \\
& solution using conventional mathe- \\
& matical techniques.
\end{tabular}

2. Data requirement

3. Handling capability of non-linearity
Conventional techniques first use the principles (e.g., physical laws) to derive the relationships of the system, which usually justifiably simplified with many assumptions and require prior knowledge about the nature of the relationships among the data.

In the real world, it is prone that complex and highly non-linear problems are encountered. If the relationship between $\mathrm{x}$ and $\mathrm{y}$ is non-
Artificial Intelligence

Techniques

Artificial intelligence (AI) is a computational method that attempts to imitate, in a very simplistic way, the human cognition capability so as to solve PMU placement problem

AI models are dependent on data that rely on the data alone to determine the structure and parameters that solve a system problem, with fewer assumptions about the physical behavior of the system.

The prior information of the nature of the non-linearity is not required for AI models. 
4. Assumptions

5. Problem solving approach

6. Up gradation of results

7. Precision \& Accuracy

8. $\quad$ Efficiency

9. Transparency \& model uncertainty

10. Prior information linear, mathematical analysis can be applied successfully only if prior knowledge of the nature of the non-linearity exists.

For most traditional mathematical models, the lack of physical understanding is usually supplemented by either simplifying the problem or incorporating several assumptions into the models.

Mathematical models rely on assuming the structure of the model in advance, which may be sub-optimal. Hence many mathematical models fail to simulate the complex engineering problems

In the conventional mathematical techniques this up gradation of the past results is not possible.

As compare to AI techniques, mathematical models not provide highest level of accuracy due to various assumptions incorporated in the modeling.

Conventional mathematical techniques require more time to solve the complex problem as compare to $\mathrm{AI}$ techniques.

In mathematical modeling such as MIP The existence of integer decision variables makes the problem hard to solved using conventional $\log$ barrier.

In mathematical techniques are the process are mathematically proved.
There is less need to either simplify the problem or incorporate assumptions in AI modals.

AI techniques are datadriven approaches in which the model development is based on training of input-output data pairs to determine the structure and parameters of the model.

AI models can always be updated to obtain better results by presenting new training examples as new data become available

Artificial intelligence techniques provide greater precision and accuracy due to less chances of error.

Artificial intelligence techniques can be utilized in carrying out repetitive and time-consuming tasks efficiently.

AI techniques are still facing classical opposition due to some inherent inadequacy that need further attention in the future including the lack of transparency, knowledge extraction, and model uncertainty

Special attention should be paid to incorporating prior knowledge about the underlying physical process based on engineering judgment or human expertise into the learning formulation. 


\section{Conclusions}

Under smart gird environment the placement of phasor measurement units is very critical because accurate measurement of active power transferred is required. Hence in this paper a comprehensive review of PMU placement methods is presented. Optimal PMU placement (OPP) problem has been solved by utilizing numerous optimization methods. To obtain solutions for the OPP problem, two major techniques including mathematical methods and heuristic algorithms exist. The correct methodology is required to find the optimal placement of PMUs in power network. The presented review of heuristic and conventional/mathematical optimization techniques could largely help researchers in terms of employing new concepts to solve the OPP problem. Further economic point can also be added to make optimal PMU placement problem more practical to the industry stakeholders. This review can be helpful to researchers for making improvement in already existing methods and develop new heuristic optimization approaches for multiobjective optimal PMU placement considering the constraints. Additionally security assessment with PMU's is also a important issue which could also be incorporated.

\section{References}

1. Beg Mohammadi M, Hooshmand RA, Haghighatdar Fesharaki F (2016) A New Approach for Optimal Placement of PMUs and Their Required Communication Infrastructure in Order to Minimize the Cost of the WAMS. In: IEEE Transactions on Smart Grid, vol 7, pp 84-93

2. Mazhari SM, Monsef H, Lesani H, Fereidunian A (2013) A Multi-Objective PMU Placement Method Considering Measurement Redundancy and Observability Value Under Contingencies. In: IEEE Transactions on Power Systems, vol 28, pp 2136-2146

3. Wen MHF, Xu J, Li VOK (2013) Optimal Multistage PMU Placement for Wide-Area Monitoring. In: IEEE Transactions on Power Systems, vol 28, pp 4134-4143

4. Bhuvana Ramachandran A, Bellarmine TG (2013) Improving observability using optimal placement of phasor measurement units. Electr Power Energy Syst 56(2014):55-63

5. Rakpenthai C, Premrudeepreechacharn S, Uatrongjit S, Watson NR (2007) An Optimal PMU Placement Method Against Measurement Loss and Branch Outage. IEEE Trans Power Delivery 22(1):101-107

6. Aminifar F, Lucas C, Khodaei A, Fotuhi-Firuzabad M (2009) Optimal Placement of Phasor Measurement Units Using Immunity Genetic Algorithm, vol 24

7. Milosevic B, Begovic M (2003) Non-dominated sorting genetic algorithm for optimal phasor measurement placement. In: IEEE Transactions on Power Systems, vol 18, pp 69-75

8. Allagui B, Aribia HB, Abdallah HH (2012) Optimal placement of Phasor Measurement Units by genetic algorithm, 2012 First International Conference on Renewable Energies and Vehicular Technology. Hammamet:434-439
9. Dehghania M, Goelb L, Lic W (2014) PMU based observability reliability evaluation in electric power systems. Electr Power Syst Res 116(2014):347-354

10. El-Zonkoly A (2006) Optimal meter placement using genetic algorithm to maintain network observability. Expert Systems with Applications 31(1):193-198

11. Gamm AZ, Kolosok IN, Glazunova AM, Korkina ES (2008) PMU placement criteria for EPS state estimation, Electric Utility Deregulation and Restructuring and Power Technologies, 2008. DRPT 2008, Third International Conference on, Nanjuing:645-649

12. Jamuna K, Swarup KS (2011) Optimal placement of PMU and SCADA measurements for security constrained state estimation. Electr Power Energy Syst 33(2011):1658-1665

13. Mousavian S, Feizollahi MJ (2015) An investment decision model for the optimal placement of phasor measurement units. Expert Systems with Applications 42(21):7276-7284

14. Müller HH, Castro CA (2013) Optimal substation PMU placement method for the two-level state estimator. Electric Power and Energy Conversion Systems (EPECS), 2013 3rd International Conference on, Istanbul:1-6

15. Sajan KS, Tyagi B (2011) Optimal placement of PMU with optimal branch current phasors for complete and incomplete observability. 2011 IEEE Power and Energy Society General Meeting, San Diego, CA, pp 1-5

16. Zhao Y, Yuan P, Ai Q, Lv T (2015) Optimal PMU placement considering topology constraints. Electr Power Energy Syst 73(2015):240-248

17. Li Q, Negi R, Ilić MD (2011) Phasor measurement units placement for power system state estimation: A greedy approach. 2011 IEEE Power and Energy Society General Meeting, San Diego, CA, pp 1-8

18. Li Q, Cui T, Weng Y, Negi R, Franchetti F, Ilic MD (2013) An Information-Theoretic Approach to PMU Placement in Electric Power Systems. IEEE Trans Smart Grid 4(1):446-456

19. Yang Y, Roy S (2013) PMUplacement for optimal three-phase state estimation performance. Smart Grid Communications (Smart Grid Comm.), 2013 IEEE International Conference on, Vancouver, BC:342-347

20. Yang P, Tan Z, Wiesel A, Nehorai A (2015) Placement of PMUs Considering Measurement Phase-Angle Mismatch. IEEE Trans Power Delivery 30(2):914-922

21. Peng C, Sun H, Guo J (2010) Multi-objective optimal PMU placement using a non-dominated sorting differential evolution algorithm. Electr Power Energy Syst 32(2010):886892

22. Hajian M, Ranjbarb AM, Amraee T, Mozafari B (2011) Optimal placement of PMUs to maintain network observability using a modified BPSO algorithm. Electr Power Energy Syst 33(2011):28-34

23. Rather ZH, Chen Z, Thø gersen P, Lund P, Kirby B (2015) Realistic Approach for Phasor Measurement Unit Placement: Consideration of Practical Hidden Costs. In: IEEE Transactions on Power Delivery, vol 30, pp 3-15

24. Su C, Chen Z (2010) Optimal Placement of Phasor Measurement Units with New Considerations. 2010 Asia-Pacific Power and Energy Engineering Conference, Chengdu, pp 1-4

25. Wang JM, Li C, Zhang J (2012) Optimal Phasor Measurement Unit Placement by an Improved PSO Algorithm. 2012 AsiaPacific Power and Energy Engineering Conference, Shanghai, pp 1-4

26. Baldwin TL, Mili L, Boisen Jr MB, Adapa R (1993) Power System Observability With Minimal Phasor Measurement Placement. IEEE Trans Power Syst 8(2):707-715 
27. Albuquerque RJ, Paucar VL (2013) Evaluation of the PMUs Measurement Channels Availability for Observability Analysis. In: IEEE Transactions on Power Systems, vol 28, pp 2536-2544

28. Hurtgen M, Maun J-C (2010) Optimal PMU placement using Iterated Local Search. Electr Power Energy Syst 32(2010):857-860

29. Liao C-S, Hsieh T-J, Guo X-C, Liu J-H, Chu C-C (2015) Hybrid search for the optimal PMU placement problem on a power grid. Eur J Oper Res 243(3):985-994

30. Qi J, Sun K, Kang W (2015) Optimal PMU placement for power system dynamic state estimation by using empirical observability Gramian. 2015 IEEE Power \& Energy Society General Meeting, Denver ,CO, pp 1-1

31. Saha Roy BK, Sinha AK, Pradhan AK (2012) An optimal PMU placement technique for power system observability. Electr Power Energy Syst 42(2012):71-77

32. Tai X, Marelli D, Rohr E, Fu M (2013) Optimal PMU placement for power system state estimation with random component outages. Electr Power Energy Syst 51(2013):35-42

33. Venkatesh $\mathrm{T}$, Jain $\mathrm{T}$ (2015) Intelligent-search technique based strategic placement of synchronized measurements for power system observability. Expert Systems with Applications 42(10):4768-4777

34. Nuqui RF, Phadke AG (2005) Phasor measurement unit placement techniques for complete and incomplete observability. In: IEEE Transactions on Power Delivery, vol 20, pp 2381-2388

35. Yang Y, Shu H, Yue L (2009) Engineering practical method for PMU placement of 2010 Yunnan power grid in China. 2009 International Conference on Sustainable Power Generation and Supply, Nanjing, pp 1-6

36. Koutsoukis NC, Manousakis NM, Georgilakis PS, Korres GN (2013) Numerical observability method for optimal phasor measurement units placement using recursive Tabu search method. In: IET Generation, Transmission \& Distribution, vol 7, pp 347-356

37. Peng J, Sun Y, Wang HF (2006) Optimal PMU placement for full network observability using Tabu search algorithm. Electr Power Energy Syst 28(2006):223-231

38. Chakrabarti S, Kyriakides E (2008) Optimal Placement of Phasor Measurement Units for Power System Observability. In: IEEE Transactions on Power Systems, vol 23, pp 1433-1440

39. Wu J, Xiong J, Shil P, Shi Y (2014) Optimal PMU placement for identification of multiple power line outages in smart grids 2014 IEEE 57th International Midwest Symposium on Circuits and Systems (MWSCAS), College Station, TX, pp 354-357

40. Gou B, Kavasseri RG (2014) Unified PMU Placement for Observability and Bad Data Detection in State Estimation, vol 29

41. Rakpenthai C, Premrudeepreechacharn S, Uatrongjit S, Watson NR (2007) An Optimal PMU Placement Method against Measurement Loss and Branch Outage," Power Engineering Society General Meeting, 2007, IEEE, Tampa, FL, pp 1-1

42. Aminifar F, Fotuhi-Firuzabad M, Safdarian A, Shahidehpour M (2014) Observability of Hybrid AC/DC Power Systems With Variable-Cost PMUs. In: IEEE Transactions on Power Delivery, vol 29, pp 345-352

43. Gomathi V, Ramachandran V (2011) Optimal location of PMUs for complete observability of power system network, Electrical Energy Systems (ICEES), 2011 1st International Conference on, Newport Beach, CA, pp. 314-317

44. Fish AA, Chowdhury S, Chowdhury SP (2011) Optimal PMU placement in a power network for full system observability, 2011 IEEE Power and Energy Society General Meeting, San Diego, CA, pp 1-8

45. Yammani C, Narsi RK, Maheswarapu S (2015) Optimal placement of PMU's considering sensitivity analysis, Advancements in Power and Energy (TAP Energy), 2015 International Conference on, Kollam, pp 40-44
46. Sodhi R, Srivastava SC, Singh SN (2011) Multi-criteria decisionmaking approach for multistage optimal placement of phasor measurement units. In: IET Generation, Transmission \& Distribution, vol 5, pp 181-190

47. Biswal A, Mathur HD (2015) Identification of optimal locations of PMUs for WAMPAC in smart grid environment. Advancements in Power and Energy (TAP Energy), 2015 International Conference on, Kollam:369-374

48. Zare J, Aminifar F, Sanaye-Pasand M (2015) SynchrophasorBased Wide-Area Backup Protection Scheme with Data Requirement Analysis. In: IEEE Transactions on Power Delivery, vol 30, pp 1410-1419

49. Thorp JS, Phadke AG, Karimi KJ (1985) Real Time VoltagePhasor Measurements for Static State Estimation. In: IEEE Power Engineering Review, vol. PER-5, no. 11, pp 32-33

50. Aminifar F, Fotuhi-Firuzabad M, Shahidehpour M, Khodaei A (2011) Probabilistic Multistage PMU Placement in Electric Power Systems, vol 26

51. Xu B, Abur A (2004) Observability analysis and measurement placement for systems with PMUs. Power Systems Conference and Exposition, 2004. IEEE PES 2:943-946

52. Abiri E, Rashidi F, Niknam T, Salehi MR (2014) Optimal PMU placement method for complete topological observabilit of power system under various contingencies. Electr Power Energy Syst 61(2014):585-593

53. Aghaei J, Baharvandi A, Rabiee A, Akbari MA (2015) Probabilistic PMU Placement in Electric Power Networks: An MILP-Based Multiobjective Model. In: IEEE Transactions on Industrial Informatics, vol 11, pp 332-341

54. Sodhi R, Srivastava SC, Singh SN (2009) Optimal PMU placement to ensure system observability under contingencies, 2009 IEEE Power \& Energy Society General Meeting, Calgary, AB:1-6

55. Dua D, Dambhare S, Gajbhiye RK, SOman SA (2008) Optimal Multistage Scheduling of PMU Placement An ILP Approach. IEEE Trans Power Delivery 23(4)

56. Kavasseri R, Srinivasan SK (2011) Joint Placement of Phasor and Power Flow Measurements for Observability of Power Systems. IEEE Trans Power Syst 26(4)

57. Rihan M, Ahmad M, Beg MS, Anees MA (2013) Robust and economical placement of phasor measurement units in Indian Smart Grid. 2013 IEEE Innovative Smart Grid Technologies-Asia (ISGT Asia) Bangalore:1-6

58. Bhonsle JS, Junghare AS (2015) An Optimal PMU-PDC Placement Technique in Wide Area Measurement System, 2015 International Conference on Smart Technologies and Management for Computing, Communication, Controls, Energy and Materials (ICSTM), India. 6 - 8 May 2015. pp 401-405

59. Abbasy NH, Ismail HM (2009) A Unified Approach for the Optimal PMU Location for Power System State Estimation, vol 24

60. Gupta N, Goyal M, Tripathy P (2012) A novel approach for optimal placement of PMUs with minimum measurement channels, Power and Energy (PECon), 2012 IEEE International Conference on, Kota Kinabalu, pp 505-509

61. Dua D, Dambhare S, Gajbhiye RK, Soman SA (2008) Optimal multistage scheduling of PMU placement: An ILP approach. IEEE Trans Power Del 23(4):1812-1820

62. Chen J, Abur A (2006) Placement of PMUs to Enable Bad Data Detection in State Estimation. In: IEEE Transactions on Power Systems, vol. 21, no. 4, pp 1608-1615

63. Razavi SE, Falaghi H, Ramezani M (2013) A new integer linear programming approach for multi-stage PMU placement. Smart Grid Conference (SGC), 2013, Tehran:119-124

64. Wang F, Zhang W, Li P (2012) Optimal incremental placement of PMUs for power system observability, 2012 IEEE Power and Energy Society General Meeting, San Diego, CA, pp 1-7 
65. Korres GN, Manousakis NM, Xygkis TC, Löfberg J (2015) Optimal phasor measurement unit placement for numerical observability in the presence of conventional measurements using semi-definite programming," in IET Generation. Transm Distrib 9(15):2427-2436

66. Kavasseri R, Srinivasa SK (2011) Joint placement of phasor and conventional power flow measurements for fault observability of power systems. IET Gene Trans Distrib 5(10):10191024

67. Nan X, Beng GH (2013) Optimal PMU placement with local redundancy of conventional measurements. Computational Intelligence Applications In Smart Grid (CIASG), 2013 IEEE Symposium on, Singapore: $1-5$

68. Fadiran JI, Chowdhury S, Chowdhury SP (2013) A multi-criteria optimal phasor measurement unit placement for multiple applications, 2013 IEEE Power \& Energy Society General Meeting, Vancouver, BC, pp 1-5

69. Putranto LM, Hara R, Kita H, Tanaka E (2014) Voltage stabilitybased PMU placement considering $\mathrm{N}-1$ line contingency and power system reliability Power Engineering and Renewable Energy (ICPERE), 2014 International Conference on, Bali, pp $120-125$

70. Gou B (2008) Generalized integer linear programming formulation for optimal PMU placement. IEEE Trans Power Syst 23(3):1099-1104

71. Zhao Y, Chen J, Goldsmith A, Poor HV (2014) Identification of Outages in Power Systems With Uncertain States and Optimal Sensor Locations. IEEE J Sel Top Sign Proces 8(6)

72. Huang L, Sun Y, Xu J, Gao W, Zhang J, Wu Z (2014) Optimal PMU Placement Considering Controlled Islanding of Power System. In: IEEE Transactions on Power Systems, vol 29, pp 742755

73. Gómez Oscar, Ríos MA (2013) ILP-based multistage placement of PMUs with dynamic monitoring constraints. Electr Power Energy Syst 53(2013):95-105

74. Azizi S, Gharehpetian GB, Dobakhshari AS (2013) Optimal Integration of Phasor Measurement Units in Power Systems Considering Conventional Measurements. In: IEEE Transactions on Smart Grid, vol 4, pp 1113-1121

75. Dehghani M, Shayanfard B, Khayatian AR (2013) PMU Ranking Based on Singular Value Decomposition of Dynamic Stability Matrix. In: IEEE Transactions on Power Systems, vol 28, pp 2263-2270
76. Esmaili M, Gharani K, Shayanfar HA (2013) Redundant Observability PMU Placement in the Presence of Flow Measurements Considering Contingencies. In: IEEE Transactions on Power Systems, vol 28, pp 3765-3773

77. Rashidia F, Abiria E, Niknamb T, Salehia MR (2015) Optimal placement of PMUs with limited number of channels for complete topological observability of power systems under various contingencies. Electr Power Energy Syst 67(2015):125-137

78. Azizi S, Dobakhshari AS, Sarmadi SAN, Ranjbar AM (2012) Optimal PMU Placement by an Equivalent Linear Formulation for Exhaustive Search. IEEE Trans Smart Grid 3(1)

79. Yuill W, Edwards A, Chowdhury S, Chowdhury SP (2011) Optimal PMU placement: A comprehensive literature review. 2011 IEEE Power and Energy Society General Meeting, San Diego, CA, pp 1-8

80. Chakrabarti S, Kyriakides E, Albu M (2009) Uncertainty in Power System State Variables Obtained Through Synchronized Measurements. IEEE Trans Instrum Meas 58(8)

81. Chakrabarti S, Kyriakides E, Eliades DG (2009) Placement of Synchronized Measurements for Power System Observability. IEEE Trans. Power Delivery 24(1)

82. Manousakis NM, Korres GN (2013) A Weighted Least Squares Algorithm for Optimal PMU Placement. IEEE Trans Power Syst 28(3)

83. Manousakis NM, Korres GN, Georgilakis PS (2012) Taxonomy of PMU Placement Methodologies. IEEE Trans Power Syst 27(2)

84. Nazari-Heris M, Mohammadi-Ivatloo B (2015) Application of heuristic algorithms to optimal PMU placement in electric power systems: An upyeard review. Renew Sust Energ Rev 50(2015):214-228

85. More KK, Jadhav HT A Literature Review on Optimal Placement of Phasor Measurement Units, 2013 International Conference on Power, Energy and Control (ICPEC):2013

86. Moarref B, Mehdipour A, Yazdanipour M (2012) Using branch and bound method and optimal placement of phasor measurement unit of power network in Khouzestan province in Iran, 2012 IEEE International Conference on Power Electronics, Drives and Energy Systems (PEDES). Bengaluru:1-6

87. Mohammadi-Ivatloo B, Hosseini SH (2008) Optimal PMU placement for power system observability considering secondary voltage control, Electrical and Computer Engineering, 2008. CCECE 2008. Canadian Conference on, Niagara Falls, ON, pp 000365000368 